\title{
Chapter 19 \\ The Need for a Holistic Approach \\ to Prevent Reproductive Cancers Among \\ US Latinas: The Potential Impact \\ of Normalizing Sexuality and Improving \\ Communication
}

Julia Lechuga and Katherine Melo

\section{Introduction}

US Latina women are disproportionately affected by cervical cancer incidence compared to non-Latina white women. The cervical cancer incidence rate among US Latinas is estimated at 9.9/100,000 compared to the 7.7/100,000 estimated rate of non-Latina white women [1]. Compared to non-Latina white women, Latinas are less likely to screen for cervical cancer, more likely to get a cervical cancer diagnosis later in the course of the disease, and less likely to receive follow-up treatment services after a positive diagnosis, leading to increased risk of mortality [2].

Research suggests that a myriad of factors, operating at multiple levels of influence, negatively influence cervical cancer screening and receipt of follow-up treatment in a timely fashion. Factors at the individual level include low knowledge about the causes of cervical cancer and forms of prevention, screening, and treatment [3] and demographic factors such as low acculturation, foreign birth, and low income and educational attainment $[4,5]$. Other factors operating at the individual level include adherence to Latino cultural beliefs and norms such as dignity, respect, fatalism, and traditional gender norms [6]. At the structural level, factors such as lack of access to health care services and logistical barriers to accessing services such as lack of transportation and childcare have been shown to affect screening and receipt of follow-up treatment $[7,8]$.

To promote cervical cancer screening and treatment, a literature review was conducted on interventions; several gaps were identified, including few interventions uniquely targeting Latinas and a dearth of interventions informed by theories explicating the manner in which culture may affect screening and treatment, such as the PEN-3

\footnotetext{
J. Lechuga $(\bowtie) \cdot$ K. Melo

Counseling Psychology Program, Lehigh University, Bethlehem, PA, USA

e-mail: jul416@lehigh.edu
} 
model of cultural influence [9]. Specifically, most cervical cancer screening promotion interventions have been informed by health-behavior change theories prominent in psychology, such as the Health Belief Model, Theory of Reasoned Action, Transtheoretical Model, Social Learning Theory, Social Cognitive Theory, and Social Influence Theory. The literature review clearly identified a need for interventions that simultaneously target factors operating at multiple levels of influence and for a broadening of focus beyond cancer screening as end outcomes to include improvements in sexual and reproductive health, broadly speaking [9]. Using a theoretical lens accounting for the complex interplay of factors affecting human behavior, a closer look at research findings and intervention efforts undertaken in other domains of Latino sexual and reproductive health may promote the development of interventions targeting multiple levels of influence, including structural-level factors beyond individual-level precursors of behavior change.

A closer look at disparities in sexual and reproductive health among Latinos indicates that Latinos experience negative health outcomes in multiple domains of sexual and reproductive health, not only cervical cancer. For example, Latinas are less likely to screen for breast cancer and are at increased risk of mortality compared to non-Latina white women [10]. Furthermore, the rate of sexually transmitted infections (STIs) including HIV and unintended teen pregnancies among Latinos is double the rate of non-Latino whites $[1,2,11]$. These statistics underscore the need to develop and test interventions aimed at targeting root causes of disparities in sexual and reproductive health.

We argue that there is the need to conduct research informed by theories which conceptualize the individual as nested in complex systems, to further understand the manner in which contextual and individual-level factors interact and influence adoption of sexual and reproductive health preventative behaviors across domains. Culture is a powerful system that influences human behavior. According to the socioecological model by Bronfenbrenner [12], culture is a macrosystem in which individuals are nested; it can be conceptualized as institutional and familial socialization practices that transmit values, norms, and behavioral expectations through generations. Past research has examined the effect of culture on various domains of sexual and reproductive health among Latinos. However, when considering how culture may influence various systems in which the individual is embedded and how it may ultimately lead to individual differences in sexual and reproductive health decision-making, there is a need to operationalize it and its effects in a more complex way. Here we present findings from two separate studies that highlight the influence of two important cultural factors operating at the macro and micro levels of influence: embarrassment and shame ascribed to sexuality (macro level) and communication about sexuality among members of a social network (micro level).

\section{Study 1}

The purpose of the first study was to understand the influence of communication about sexuality in adoption of the HPV vaccine-a three-dose vaccine recommended for children and pre-adolescents between the ages of 11 and 12. Catch-up vaccination is recommended for adolescents who were not vaccinated at the recommended 
age. Our study sample consisted of 65 mother-daughter dyads who self-identified as Latino. We purposefully sampled $50 \%$ of daughters who had received at least one dose of the HPV vaccine and employed a mixed methods qualitative dominant sequential design.

\section{Materials, Procedure, and Approach to Analysis}

Participants were recruited from a variety of venues including churches, schools, and supermarkets. Mothers and daughters were interviewed separately. Mother was asked to sign an informed consent form for her and daughter, and daughter was assented separately. Participants answered open-ended questions as part of the qualitative portion of the study followed by a brief demographic survey, which also assessed whether mother had engaged in the discussions about sexuality with daughter and the specific sexuality-related topics discussed. We content analyzed open-ended responses, computed chi-square tests of association, and performed a logistic regression analysis. We computed a chi-square test to assess the association between having engaged in discussions about sexuality with daughter and vaccination uptake. We computed a stepwise blocked logistic regression to understand which sexuality-related topics were significantly associated with vaccination uptake. In the first block of the regression equation, we entered the daughters' age. In the second block we entered specific sexuality-related topics using the stepwise procedure. Our goal was to assess which of these variables would emerge as significantly associated with vaccination uptake.

\section{Results}

A larger proportion of mothers who had vaccinated their daughters (64\%) reported to have engaged in discussions about sexuality with daughter compared to mothers who had not vaccinated $(36 \%)\left(\chi^{2}=5.38, p=0.02\right)$. Content analysis of the responses suggested that the content of sexuality-related discussions differed between those who had and had not vaccinated. Specifically, the content of discussions between mothers and their daughters who had been vaccinated seemed to encompass a variety of topics related to sexual health including anatomy, puberty, appropriate timing of initiation of sexual relations, and the importance of engaging in behaviors to protect oneself against STIs. In contrast, the majority of mothers of daughters who had not been vaccinated conveyed feeling uncomfortable and embarrassed about speaking with daughter about sexual health, and the content of their communication was value-based including admonishing daughter about the inappropriateness of initiating sexual relations before marriage. Table 19.1 presents the results of the last step of the logistic regression analysis. Daughters' age emerged as a significant predictor of uptake; the older the daughter the more likely she is to be vaccinated. Furthermore, engaging in discussions about birth control emerged as significantly associated with uptake, predicting $40 \%$ of the variance in vaccination uptake. 
Table 19.1 Factors associated with vaccination uptake

\begin{tabular}{l|l|l|l|l|l|l}
\hline Variable & B & SE & OR & $95 \%$ CI & $\begin{array}{l}\text { Wald } \\
\text { statistic }\end{array}$ & $p$ \\
\hline Daughter's age & 0.50 & 0.21 & 1.65 & $1.09,2.50$ & 5.68 & 0.01 \\
\hline Talk birth control & 1.71 & 0.77 & 5.56 & $1.21,25.50$ & 4.87 & 0.02 \\
\hline
\end{tabular}

Note: Data is for mothers $(N=65)$. Talk birth control = extent to which mothers reported having discussed birth control methods with daughters. Talking about birth control with daughters alone predicted $40 \%$ of the variance in vaccination uptake

\section{Study 2}

The purpose of the second study was to understand the sociocultural factors associated with attitudes toward cancer screening in a sample of US Latinas. A crosssectional survey was administered to a sample of 85 US Latinas as part of a larger study designed to test the effect of a community-based, participatory, sexual-health intervention delivered by community health workers.

\section{Materials, Procedure, and Approach to Analysis}

Community health workers (CHWs) recruited participants in locations where Latinos congregate regularly (e.g., supermarkets and churches). To be eligible to participate, participants had to be 18 years old and self-identify as Latina. Participants who volunteered to participate were asked to answer a survey asking demographic questions including age, household income, and whether they had ever had a Pap smear test and/ or mammogram if eligible. The survey also included questions assessing negative attitudes toward cancer screening and sociocultural constructs such as shame and embarrassment ascribed to sexuality. To understand whether sociocultural factors such as shame and embarrassment ascribed to sexuality would emerge as significant predictors of attitudes toward cancer screening, we computed a hierarchical multivariate linear regression analysis. In the first block of the equation we entered demographic variables including age, income, and whether the participant had ever had a Pap smear test and/ or mammogram using the enter procedure. In the second block of the regression equation, we entered sociocultural variables using the stepwise procedure.

\section{Results}

Table 19.2 presents the results of the last step of the regression model. The sociocultural variable shame and embarrassment ascribed to sexuality was significantly associated with negative attitudes toward cancer screening above and beyond demographic variables typically associated with access to health care and predicted an additional $30 \%$ of variability in negative attitudes toward cancer screening. 
Table 19.2 Factors associated with negative attitudes toward cancer screening

\begin{tabular}{l|l|l|l|l|l}
\hline Variable & $B$ & SE & $\beta$ & $95 \%$ CI & $T$ \\
\hline Age & -0.03 & 0.02 & -0.35 & $-0.07,0.01$ & -1.53 \\
\hline Household income & 0.00 & 0.00 & -0.08 & $-0.01,0.00$ & -0.48 \\
\hline Ever had a pap test & 0.38 & 0.45 & 0.16 & $-0.56,1.33$ & 0.83 \\
\hline Ever had a mammogram & 0.81 & 0.46 & 0.35 & $-0.12,1.74$ & 1.77 \\
\hline Shame and discomfort with sexuality & 0.54 & 0.18 & 0.52 & $0.16,0.92$ & 2.92 \\
\hline
\end{tabular}

Note: Data is for Latina women $(N=85)$. The addition of shame and discomfort with sexuality predicted an additional $30 \%$ of the variance in negative attitudes toward cancer screening

\section{Discussion}

Operating at multiple levels of influence, culture is a key factor to be considered in order to move the field forward and reduce Latino health disparities in sexual and reproductive health broadly speaking. Our argument is put forth in light of several factors. First, the majority of the prominent behavior change theories which have dominated health promotion and intervention research rest on the assumption that the most powerful determinants of behavior are personally derived attitudes and cognitions about the health behavior in question, such as perceived benefits and consequences of the behavior [13]. This assumption is questionable as research with individuals who conceptualize the self as an extension of the in-group (i.e., Latinos) compared with individuals who conceptualize the self as separate from others (i.e., non-Latino whites) [14] suggests that the function of behavior is to fulfill social obligations [15]; and self-efficacy is more the product of support from important members of the social network than volitional control [16]. Second, sexual and reproductive health behaviors around the world are heavily influenced by societal level norms and expectations; consequently, developing interventions that target multiple levels of influence with culture considered as a central piece could yield interventions that target root causes of disparities and yield sustainable behavior change.

\section{References}

1. American Cancer Society. Cancer facts \& figures for Hispanics/Latinos 2015-2017. Atlanta: American Cancer Society; 2015.

2. American Cancer Society. Cancer facts \& figures for Hispanics/Latinos 2012-2014. Atlanta: American Cancer Society; 2012.

3. Otero-Sabogal R, Stewart S, Sabogal F, Brown BA, Perez-Stable EJ. Access and attitudinal factors related to breast and cervical cancer rescreening: why are Latinas still underscreened? Health Educ Behav. 2003;30(3):337-59. https://doi.org/10.1177/109019810303000-3008.

4. Garces-Palacio IC, Scarinci IC. Factors associated with perceived susceptibility to cervical cancer among Latina immigrants in Alabama. Matern Child Health J. 2012;16(1):242-8. https://doi.org/10.1007/s10995-010-0737-x. 
5. Shah M, Zhu K, Wu H, Potter J. Hispanic acculturation and utilization of cervical cancer screening in the US. Prev Med. 2006;42(2):146-9. https://doi.org/10.1016/j.ypmed.2005.10.002.

6. Arredondo EM, Pollak K, Costanzo PR. Evaluating a stage model in predicting monolingual spanish-speaking Latinas' cervical cancer screening practices: the role of psychosocial and cultural predictors. Health Educ Behav. 2008;35(6):791-805. https://doi. org/10.1177/109019-8107303250.

7. Scarinci IC, Beech BM, Kovach KW, Bailey TL. An examination of sociocultural factors associated with cervical cancer screening among low-income Latina immigrants of reproductive age. J Immigr Health. 2003;5(3):119-28.

8. White K, Garces IC, Bandura L, McGuire AA, Scarinci IC. Design and evaluation of a theorybased, culturally relevant outreach model for breast and cervical cancer screening for Latina immigrants. Ethn Dis. 2012;22(3):274-80.

9. Mann L, Foley KL, Tanner AE, Sun CJ, Rhodes SD. Increasing cervical cancer screening among US Hispanics/Latinas: a qualitative systematic review. J Cancer Educ. 2015;30(2):374-87.

10. Nahleh Z, Otoukesh S, Mirshahidi H. Disparities in breast cancer: a multi-institutional comparative analysis focusing on American Hispanics. Cancer Med. 7(6):2710-7.

11. Mosher WD, Jones J, Abma JC. Intended and unintended births in the United States: 19822010. Natl Health Stat Report. 2012;(55):1-28.

12. Bronfenbrenner U. The ecology of human development: experiments by nature and design. Cambridge: Harvard University Press; 1979.

13. Rosenstock IM. Why people use health services. Milbank Mem Fund Q. 1966;44(3):1107-8.

14. Oyserman D, Coon HM, Kemmelmeier M. Rethinking individualism and collectivism: evaluation of theoretical assumptions and meta-analyses. Psychol Bull. 2002;128(1):3-72.

15. Riemer H, Shavitt S, Koo M, Markus HR. Preferences don't have to be personal: expanding attitude theorizing with a cross-cultural perspective. Psychol Rev. 2014;121(4):619-48.

16. Pasick RJ, Burke NJ. A critical review of theory in breast cancer screening promotion across cultures. Annu Rev Public Health. 2008;29:351-68.

Open Access This chapter is licensed under the terms of the Creative Commons AttributionNonCommercial 4.0 International License (http://creativecommons.org/licenses/by-nc/4.0/), which permits any noncommercial use, sharing, adaptation, distribution and reproduction in any medium or format, as long as you give appropriate credit to the original author(s) and the source, provide a link to the Creative Commons license and indicate if changes were made.

The images or other third party material in this chapter are included in the chapter's Creative Commons license, unless indicated otherwise in a credit line to the material. If material is not included in the chapter's Creative Commons license and your intended use is not permitted by statutory regulation or exceeds the permitted use, you will need to obtain permission directly from the copyright holder. 